\title{
Regeneración de Sebastiania commersoniana (Euphorbiaceae) EN RELACIÓN AL GANADO Y LA DISTANCIA AL BOSQUE EN LAS SIERRAS DEL CENTRO de ARgentina
}

\author{
IVÁN BARBERÁ 1 , DANIEL RENISON ${ }^{1,2,3}$ y ROMINA C. TORRES R $^{1,2,3}$
}

\begin{abstract}
Summary: Regeneration of Sebastiania commersoniana (Euphorbiaceae) in relation to livestock and distance from forest in the mountains of central Argentina. Understanding the regeneration requirements of native woody species is important to guide forest conservation and restoration activities. We evaluated the regeneration niche of Sebastiania commersoniana in relation to vegetation, soil and light comparing microsites with and without seedling in two paddocks with high and low livestock density and at three distances from the edges of forest patches dominated by the species: $-2 \mathrm{~m}$ (inside the forest), $1 \mathrm{~m}$ and $3 \mathrm{~m}$ (outside the forest). We also analyzed seedling density and proportion of browsed stems. The seedlings were found in microsites with abundant herbaceous vegetation and low soil compaction. Seedling density was $134 \%$ higher with low livestock density than with high livestock density outside the forest, showing no difference inside, and decreased abruptly with increasing distance from forest edge. The proportion of browsed stems was 13 times higher with high livestock density than with low livestock density. We conclude that establishment may be favored at microsites with conserved vegetation and soil; that seedling recruitment may be restricted to the forest patches proximity; and that reduction of livestock density may promote regeneration of $\mathrm{S}$. commersoniana.
\end{abstract}

Key words: Degradation, herbivory, native forests, regeneration niche, seedling.

Resumen: Entender los requerimientos de regeneración de especies leñosas es importante para guiar prácticas de conservación y restauración de los bosques. Evaluamos el nicho de regeneración de Sebastiania commersoniana en relación a la vegetación, suelo y luminosidad comparando micrositios con y sin renoval en dos potreros con densidad de ganado alta y baja y a tres distancias del borde de los parches de bosque dominados por la especie: $-2 \mathrm{~m}$ (dentro del bosque), $1 \mathrm{~m}$ y $3 \mathrm{~m}$ (fuera del bosque). También evaluamos la densidad de renovales y la proporción de tallos ramoneados. Los renovales se encontraron en micrositios con abundante vegetación herbácea y suelo poco compactado. La densidad de renovales fue un $134 \%$ mayor con baja densidad de ganado que con alta fuera del bosque, sin diferencias dentro, y disminuyó marcadamente al aumentar la distancia al borde. La proporción de tallos ramoneados fue 13 veces mayor ante alta densidad de ganado que ante baja. Concluimos que el establecimiento puede verse favorecido en micrositios con vegetación y suelo conservados; que el reclutamiento de renovales puede encontrarse limitado a las cercanías de los parches de bosque, y que la reducción en densidad de ganado puede favorecer la regeneración de la especie.

Palabras clave: Degradación, herbivoría, bosques nativos, nicho de regeneración, renoval.

\footnotetext{
${ }^{1}$ Universidad Nacional de Córdoba, Facultad de Ciencias Exactas, Físicas y Naturales, Centro de Ecología y Recursos Naturales Renovables, Av. Vélez Sarsfield 1611, X5016GCA Córdoba, Argentina, ivanbarbera93@gmail.com

2 Instituto de Investigaciones Biológicas y Tecnológicas (IIByT-CONICET-Universidad Nacional de Córdoba), Av. Vélez Sarsfield 1611, X5016GCA Córdoba, Argentina, drenison@conicet.gov.ar, romy.ce2008@gmail.com

${ }^{3}$ ONG Ecosistemas Argentinos
} 


\section{INTRODUCCIÓN}

Una vez superados los filtros a la dispersión, las características de los micrositios a los que llegan los propágulos pueden resultar determinantes para la germinación y el establecimiento de los renovales de especies arbóreas (Fenner \& Thompson, 2005). Por esta razón, los renovales (plántulas y juveniles pequeños) frecuentemente se encuentran en ciertos tipos de micrositios (Christie \& Armesto, 2003; Baier et al., 2007), cuyas características definen el nicho de regeneración de una especie (sensu Poorter, 2007). La caracterización de los micrositios donde se encuentran los renovales de una especie puede servir como una primera aproximación al entendimiento de los factores microambientales que regulan su regeneración.

Las condiciones de vegetación y suelo de un micrositio pueden afectar la regeneración temprana controlando muchas variables importantes para la germinación y la supervivencia de renovales, tales como la disponibilidad de luz, agua y nutrientes, la temperatura y la exposición a la herbivoría (Heinemann et al., 2000; Vincent \& Davies, 2003; Munier et al., 2010; Torres \& Renison, 2016; Collin et al., 2017). Particularmente, la vegetación puede tener un efecto negativo sobre el establecimiento de especies leñosas al competir por recursos con renovales de árboles, pero también puede facilitarlo de forma directa o indirecta. La facilitación directa incluye acumulación de semillas, disminución del estrés abiótico y modificación de la disponibilidad de recursos, mientras que la facilitación indirecta puede observarse como protección ante el ramoneo o pisoteo de herbívoros o atracción de polinizadores y dispersores de semillas (Flores \& Jurado, 2003; Filazzola \& Lortie, 2014). En general, la facilitación directa es frecuente en ambientes sometidos a estrés abiótico, como los bosques estacionalmente secos (Gómez-Aparicio, 2009).

A su vez, las características de micrositio arriba mencionadas y las limitaciones a la regeneración de las especies leñosas pueden ser altamente variables en sitios que difieren en densidad de ganado doméstico (Torres \& Renison, 2015), así como en sitios donde se alternan parches de bosques y otras fisonomías de vegetación (Duncan \& Duncan, 2000; Kellner \& Swihart, 2016). Por un lado, el ganado doméstico puede disminuir la tasa de reclutamiento mediante el ramoneo y pisoteo (Griscom et al., 2009) y restringir el establecimiento de renovales de árboles a los micrositios menos degradados, resultando en una disminución de la densidad de renovales (Torres et $a l ., 2008)$. Sin embargo, la regeneración de algunas especies arbóreas puede verse favorecida por el ganado doméstico cuando el mismo dispersa sus semillas y/o reduce la competencia entre renovales y otros tipos de vegetación (Bartuszevige \& Endress, 2008; Khishigjargal et al., 2013). Por otro lado, dado que los renovales pueden encontrarse más expuestos a factores externos fuera del bosque (Davies-Colley et al., 2000), los filtros para el establecimiento pueden variar con la distancia al borde del mismo. Particularmente en sistemas estacionalmente secos, el estrés hídrico puede ser mayor fuera del bosque, debido a una mayor evapotranspiración (Holmgren et al., 1997; Caylor et al., 2005). Además, el reclutamiento de renovales puede variar con la distancia al borde del bosque debido a diferencias en la presión de herbivoría (Meiners \& Martinkovic, 2002) y a cambios en la lluvia de semillas, por encontrarse los árboles semilleros dentro del bosque (Cubiña \& Aide, 2001).

Ante la marcada disminución de áreas boscosas que muestran las regiones tropicales y subtropicales de Sudamérica en las últimas décadas (Hansen et al., 2013), el estudio de la regeneración de especies arbóreas cobra elevada importancia en esta región. En el Gran Chaco, el área de bosques secos estacionales ha sufrido una reducción anual del 2,2 \% hacia fines del siglo XX y en las Sierras de Córdoba, en el centro de Argentina, queda menos del $12 \%$ del área cubierta por bosques nativos maduros (Zak et al., 2004). Para guiar prácticas de conservación y restauración de estos ecosistemas, es importante conocer los requerimientos para la regeneración de distintas especies leñosas nativas.

El objetivo de este estudio fue realizar una primera aproximación al entendimiento de los factores que regulan la regeneración temprana de Sebastiania commersoniana (Baill.) L.B. Sm. y Downs. Esta es una especie pionera, distribuida en Sudamérica subtropical y de particular interés para la restauración de ecosistemas riparios y serranos, ya que abunda en las cercanías de los cursos de agua (Piaggio \& Delfino, 2009) y en ecosistemas serranos, donde también se la encuentra en sitios alejados de los cursos de agua (Valfré-Giorello et 
al., 2012). En el presente trabajo nos propusimos responder las siguientes preguntas en relación al nicho de regeneración de la especie: ¿Se encuentran los renovales de $S$. commersoniana asociados a micrositios con características particulares de vegetación, suelo y/o luminosidad? ¿Varía dicha asociación entre renovales y micrositios particulares según la densidad de ganado y la distancia al borde del bosque? En cuanto a la vegetación, nos planteamos las siguientes hipótesis y predicciones: (I) si predomina la competencia, esperamos encontrar renovales en micrositios con escasa vegetación; (II) si predomina la facilitación indirecta (protección ante herbivoría), esperamos encontrarlos en micrositios con abundante vegetación pero presentando una asociación más marcada ante alta densidad de ganado, y (III) si predomina la facilitación directa, esperamos encontrarlos en micrositios con abundante vegetación independientemente de la densidad de ganado. Además, en este último escenario, bajo la hipótesis de que la facilitación directa opera mediante la disminución del estrés hídrico, esperamos que la asociación de renovales con micrositios con abundante vegetación sea más notoria a mayor distancia del borde del bosque. Por otro lado, nos propusimos explorar posibles efectos del ganado y la lluvia de semillas sobre la regeneración comparando la densidad de renovales y la intensidad de ramoneo ante densidad de ganado alta y baja y a distintas distancias del borde del bosque. Bajo la hipótesis de que el ganado tiene un efecto negativo sobre la regeneración, esperamos encontrar mayor densidad de renovales ante baja densidad de ganado; en caso de existir un efecto positivo del ganado, esperamos encontrar mayor densidad de renovales ante alta densidad de ganado.

\section{Materiales y Métodos}

\section{Especie de estudio}

Sebastiania commersoniana es un árbol de entre cuatro y diez metros de altura, de copa alargada y ramas espinescentes, distribuido en Brasil, Paraguay, Uruguay y Argentina. Se la considera una especie heliófila y pionera, generalmente asociada a cursos de agua. Sus frutos presentan dehiscencia explosiva con dispersión de corto alcance y sus semillas presentan dispersión secundaria por hormigas (Demaio et al., 2002). Resulta poco probable que esta especie forme banco de semillas debido a la rápida pérdida de viabilidad que presentan las mismas (Medeiros \& Zanon, 1998). Además, presenta reproducción agámica por raíz (Valfré-Giorello et al., 2012). En un estudio realizado en Brasil, De Souza y colaboradores (2010) encontraron menor frecuencia relativa de juveniles en parches de bosque rodeados por pasturas con uso ganadero que en parches rodeados por plantaciones de eucaliptos, por lo que señalan que el ganado doméstico puede tener un efecto negativo sobre la regeneración de la especie. En las Sierras de Córdoba es frecuente encontrar ejemplares ramoneados y además se ha registrado que es una especie rebrotante (observación personal).

\section{Área de estudio}

El estudio se llevó a cabo entre los meses de junio y septiembre de 2016, en cercanías de la localidad de Cuesta Blanca, Córdoba, Argentina ( $31^{\circ} 27^{\prime} 43^{\prime \prime}$ 31 '28'25" S, 64³7'18" - 64³5'51" O; $900 \mathrm{msnm).}$ Esta área pertenece al distrito Chaqueño Serrano de la provincia fitogeográfica Chaqueña (Cabrera, 1971), y se caracteriza por presentar bosques con un dosel cerrado a altitudes bajas y en zonas protegidas como quebradas; mientras que a altitudes elevadas y en potreros expuestos y disturbados predominan los bosques abiertos y pastizales. La comunidad madura del Chaco Serrano o Bosque Serrano está dominada por Lithraea molleoides y Schinopsis lorentzii (Giorgis et al., 2011). El clima es templado cálido a subtropical, con una temperatura anual media de $13,9{ }^{\circ} \mathrm{C}$. La precipitación anual media entre 1996 y 2012 fue de $850 \mathrm{~mm}$, concentrada en los meses cálidos, entre septiembre y marzo (Colladon \& Pazos, 2014).

El estudio tuvo lugar en dos potreros contiguos de topografía similar pero con diferente densidad de ganado. El potrero con mayor densidad de ganado (135 ha; de ahora en más mencionado como "alta densidad de ganado") en las dos últimas décadas tuvo una densidad relativamente constante de 0,40 $\mathrm{UG} \mathrm{ha}^{-1}(\mathrm{UG}=$ unidades de ganado; valor reportado por el arrendatario), mientras que en las transectas de estudio estimamos por conteo de heces una densidad de 0,24 UG.ha-1 usando la metodología desarrollada por Von Müller y colaboradores (2012). El potrero con menor densidad de ganado (124 ha; de ahora en más mencionado como "baja densidad 
de ganado") también tuvo una densidad de 0,40 UG.ha $^{-1}$ (reportado por el arrendatario) al menos hasta el año 2012. Antes de agosto de 2013 se retiró el ganado de este potrero, ingresando únicamente animales pertenecientes a potreros vecinos. A partir de julio de 2015 nuevos propietarios avocan el potrero a la conservación del bosque y reportan presencia de algunos animales de forma esporádica que ingresan de los potreros vecinos debido a fallas en los alambrados y son echados ni bien son detectados. Los nuevos propietarios estiman una densidad de 0,03 UG.ha-1 ${ }^{-1}$ con ayuda de cámaras trampa y por observaciones personales, mientras que nuestras estimaciones por conteo de heces en las transectas de estudio arrojan un valor de 0,05 UG.ha ${ }^{-1}$. Según los nuevos propietarios, en julio de 2015 el potrero con baja densidad de ganado ya presentaba mayor biomasa del estrato herbáceo que el potrero con alta densidad de ganado. Dado que esta diferencia también se observaba a ambos lados de la cerca que divide los potreros, ante las mismas condiciones ambientales, ellos adjudican la diferencia en biomasa herbácea a la diferencia en densidad de ganado. La cobertura boscosa total del área de estudio se estima cercana a un $30 \%$. En toda la zona S. commersoniana presenta gran abundancia, incluso lejos de cursos de agua, encontrándose numerosos parches de bosque dominados por esta especie, frecuentemente acompañados de algunos ejemplares de L. molleoides y presentando muy baja abundancia de otras especies leñosas. Fuera de los parches de bosque estudiados son muy escasos los individuos medianos o adultos de $S$. commersoniana y de otras especies leñosas; allí abundan pastos de crecimiento en mata $y$, en el potrero con alta densidad de ganado, céspedes de pastoreo. Las deposiciones de ganado indican que los animales pasan la misma fracción de tiempo dentro y fuera de los parches de bosque de $S$. commersoniana.

\section{Diseño de muestreo}

Utilizando Google Earth se seleccionaron 18 parches de bosque dominados por S. commersoniana en cada potrero, separados por una distancia mínima de $50 \mathrm{~m}$, en los cuales la cobertura de copas de la especie fue mayor al $80 \%$ (estimada visualmente). Se procuró que cada parche tuviera un equivalente en el otro potrero en cuanto a orientación, pendiente, posición topográfica y altitud. El tamaño de los parches fue de $280 \pm 121 \mathrm{~m}^{2}$ (media \pm desvío estándar), sin diferir significativamente entre potreros (tamaño estimado con polígonos realizados con Google Earth con verificación a campo). Por cada parche se establecieron tres distancias al borde del bosque: (I) a $-2 \mathrm{~m}$ del borde (dentro del bosque); (II) a $1 \mathrm{~m}$ del borde (fuera del bosque), y (III) a $3 \mathrm{~m}$ del borde (fuera del bosque). El borde de los parches de bosque fue definido por el límite de las copas de ejemplares de $S$. commersoniana mayores a $2 \mathrm{~m}$ de altura. Fuera del bosque se consideraron dos distancias al borde para contemplar el cambio gradual en las condiciones ambientales y la disminución en lluvia de semillas con respecto al interior del bosque. Las cortas distancias al borde seleccionadas responden al abrupto cambio en coberturas arbórea y herbácea que se da entre el interior y el exterior del bosque, a la marcada disminución en densidad de renovales observada al alejarse del borde del bosque (basada en muestreos de prueba) y a la baja capacidad de dispersión que presenta la especie de estudio (hasta aproximadamente cuatro metros sin considerar dispersión secundaria; observación personal). Además, las cortas distancias escogidas permiten analizar el nicho de regeneración sin que el establecimiento se encuentre fuertemente limitado por semillas.

Para evaluar la densidad de renovales y la intensidad de ramoneo, en cada distancia al parche de bosque se estableció una transecta paralela al borde ( 3 distancias al borde $\times 18$ parches de bosque $\times 2$ potreros $=108$ transectas). En cada transecta se establecieron cuadrantes de $50 \mathrm{~cm} \times 50 \mathrm{~cm}$ dejando aproximadamente un metro de separación entre ellos (Fig. 1). Las transectas correspondientes a distintas distancias al borde del bosque variaron en su número de cuadrantes: $10 \mathrm{a}-2 \mathrm{~m}$ del borde, 20 a $1 \mathrm{~m}$ del borde y 40 a $3 \mathrm{~m}$ del borde. De esta manera se redujo la probabilidad de no encontrar renovales en las transectas que presentaban menor lluvia de semillas por encontrarse más alejadas del bosque. En cada cuadrante se registró la altura, proporción de tallos ramoneados y presencia de cotiledones de todos los renovales de $S$. commersoniana de altura $\leq 30 \mathrm{~cm}$. No se consideraron los ejemplares que sólo presentaban cotiledones, ya que no era posible diferenciarlos de las plántulas de $L$. molleoides, cuyos cotiledones son muy similares a los de la especie en estudio. Con el fin de estimar 


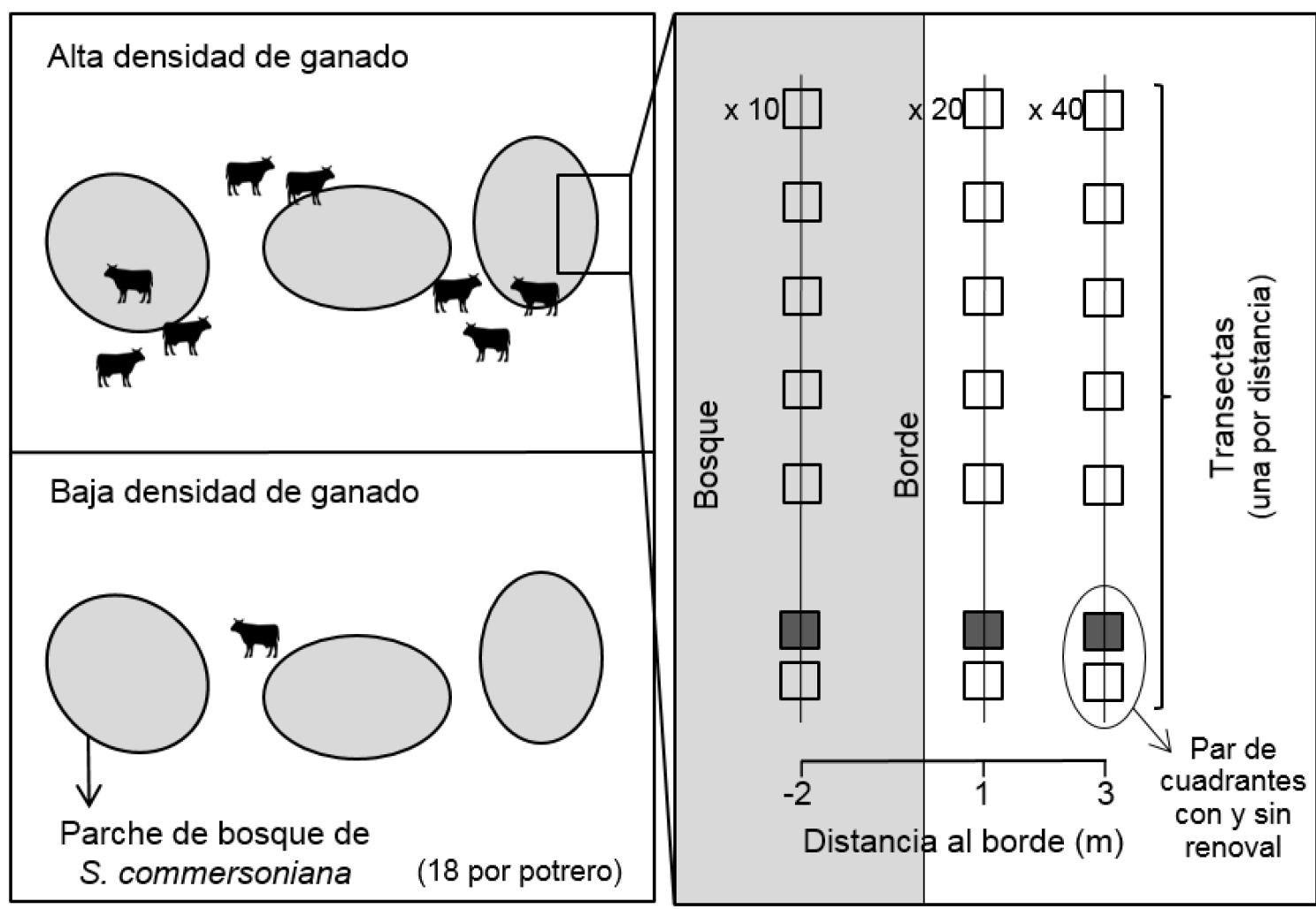

Fig. 1. Esquema del diseño de muestreo.

la importancia relativa de la reproducción agámica (por raíz), se desenterraron parcialmente los tres primeros renovales encontrados en cuadrantes separados de cada transecta que no presentaran cotiledones.

Para evaluar el nicho de regeneración de $S$. commersoniana en cuanto a condiciones de vegetación, suelo y luminosidad y su variación en función de la densidad de ganado y distancia al borde del bosque, por cada transecta se seleccionó un par de cuadrantes de $50 \mathrm{~cm} \times 50 \mathrm{~cm}$ con y sin presencia de renoval $[3$ distancias $(=$ transectas $) \times$ 18 parches de bosque $\times 2$ potreros $=108$ pares de cuadrantes; Fig. 1]. El cuadrante con renoval fue centrado en el primer renoval nacido de semilla encontrado a lo largo de cada transecta. El cuadrante sin renoval fue ubicado arrojando un cuadrante en la dirección de la transecta, a una distancia de entre 50 y $150 \mathrm{~cm}$ del cuadrante con renoval y en el sentido indicado por las agujas de un reloj, aceptándose el micrositio si no presentaba ningún renoval. En ambos cuadrantes se registraron las siguientes características del micrositio: cobertura de vegetación, suelo desnudo y roca ( $\%$ estimado visualmente); altura $(\mathrm{cm})$ de la vegetación viva y muerta en pie, menor a $2 \mathrm{~m}$ de altura y nacida en el cuadrante, medida en 10 puntos alrededor del centro del cuadrante; compactación superficial del suelo en los primeros $5 \mathrm{~mm}\left(\mathrm{Kg} . \mathrm{cm}^{-2}\right)$, determinada con un penetrómetro portátil (DGSI S-170, EEUU) en 10 puntos alrededor del centro del cuadrante; pendiente $\left({ }^{\circ}\right)$, determinada con un clinómetro (RECTA DP 2, Finlandia), y luminosidad $\left({ }^{\circ}\right)$. La luminosidad (L) fue calculada de la siguiente manera: $\mathrm{L}=\mathrm{I}^{*}(1-\mathrm{C})$, donde $\mathrm{I}$ es la insolación $\left({ }^{\circ}\right)$ y $\mathrm{C}$ es la proporción de la insolación interceptada por copas de árboles o arbustos, estimada visualmente. Para estimar la insolación se consideró el recorrido solar en los equinoccios a $-31^{\circ}$ de latitud: de Este a Oeste $\left(90^{\circ}\right.$ y $270^{\circ}$ respecto al Norte) formando un ángulo de $59^{\circ}$ con respecto al Norte $\left(0^{\circ}\right)$ en su punto más alto (Goswami et al., 2000). La 
insolación fue determinada evaluando cuántos grados del recorrido solar $\left(180^{\circ}\right)$ se encontraban obstruidos por laderas o rocas al Este y al Oeste: $\mathrm{I}=$ $180^{\circ}$ - grados obstruidos al Este - grados obstruidos al Oeste (adaptado de Renison et al., 2004).

\section{Análisis de datos}

Densidad de renovales e intensidad de ramoneo

La densidad de renovales y la proporción de tallos ramoneados fueron analizadas con Modelos Lineales Generalizados Mixtos (MLGM), empleando como variables predictoras categóricas de efectos fijos la densidad de ganado (dos niveles), la distancia al borde del bosque (tres niveles) y la interacción densidad de ganado $\times$ distancia al borde del bosque (seis niveles). La densidad de renovales (renovales. $\mathrm{m}^{-2}$ ) se modeló con distribución Binomial Negativa por tratarse de un conteo con sobredispersión (O’Hara \& Kotze, 2010; Di Rienzo et al. 2017b). Dado que esta distribución corresponde a variables discretas, se consideró como variable respuesta el número de renovales por transecta y se declaró el logaritmo natural del área de cada transecta $\left(\mathrm{A}_{t}\right)$ como variable compensatoria (offset): $\mathrm{A}_{\mathrm{t}}=\operatorname{Ln}\left(\mathrm{C}^{*} 0,25\right)$, donde $\mathrm{C}$ es el número de cuadrantes de cada transecta y 0,25 es el área de cada cuadrante en $\mathrm{m}^{2}$. De esta forma, los resultados se expresaron en términos de densidad de renovales (Di Rienzo et al., 2017b). Se incluyó el factor parche de bosque con efecto aleatorio. La proporción de tallos ramoneados de cada renoval se modeló con distribución Binomial (Bolker et al., 2008), incluyendo efecto aleatorio de la transecta y el parche de bosque de forma anidada. La altura de los renovales se incluyó como covariable, ya que los renovales más altos presentaban una mayor proporción de tallos ramoneados.

\section{Nicho de regeneración}

Para cada variable de micrositio se realizó una comparación pareada entre cuadrantes con y sin renoval. Para cada par de cuadrantes se calculó la diferencia: cuadrante con renoval - cuadrante sin renoval. En una primera instancia se analizó si la diferencia media de cada variable variaba con respecto a la densidad de ganado y/o la distancia al borde del bosque mediante Modelos Lineales Generales Mixtos. Se emplearon como variables respuesta las variables diferencia, como predictoras categóricas de efectos fijos la densidad de ganado, la distancia al borde del bosque y la interacción densidad de ganado $\times$ distancia al borde del bosque y se incluyó efecto aleatorio del factor parche de bosque cuando este aumentaba el $\mathrm{R}^{2}$ del modelo (Tabla 1).

En una segunda instancia se evaluó si la diferencia media de cada variable (total o por nivel de densidad de ganado y distancia al borde del bosque) era positiva o negativa y significativamente distinta de cero. Para las variables diferencia que no variaron en función de la densidad de ganado ni de la distancia al borde del bosque (independientemente de su signo y magnitud) se realizó una comparación entre cuadrantes con y sin renoval empleando la prueba de Wilcoxon para muestras pareadas (utilizando las variables

Tabla 1. Análisis estadísticos de las variables diferencia. MLGM = Modelo Lineal General Mixto; MLG = Modelo Lineal General.

\begin{tabular}{|c|c|c|c|c|}
\hline Variable diferencia & Modelo & $\begin{array}{l}\text { Transformación de } \\
\text { la variable original }\end{array}$ & $\begin{array}{c}\text { Factor de efecto } \\
\text { aleatorio }\end{array}$ & $\begin{array}{c}\text { Factor } \\
\text { heterocedástico }\end{array}$ \\
\hline Cobertura de vegetación (\%) & MLGM & - & Parche de bosque & Distancia al borde \\
\hline Altura de vegetación (cm) & MLGM & $\operatorname{Ln}(x+1)$ & Parche de bosque & Densidad de ganado \\
\hline Porcentaje de suelo desnudo (\%) & MLGM & $\operatorname{Ln}(x+1)$ & Parche de bosque & Densidad de ganado \\
\hline Compactación del suelo $\left(\mathrm{Kg} \cdot \mathrm{cm}^{-2}\right)$ & MLG & $\sqrt{ } x$ & - & Densidad de ganado \\
\hline Pendiente $\left(^{\circ}\right)$ & MLGM & - & Parche de bosque & - \\
\hline Porcentaje de roca $(\%)$ & ANOVA & $\operatorname{Ln}(x+1)$ & - & - \\
\hline Luminosidad $\left(^{\circ}\right)$ & Kruskal-Wallis & - & - & - \\
\hline
\end{tabular}


originales y sin calcular la diferencia). Para las variables diferencia que sí variaron en función de la densidad de ganado y/o de la distancia al borde del bosque se evaluó el intervalo de confianza del $95 \%$ para la media en cada nivel, considerando la media significativamente distinta de cero cuando el intervalo no incluía este valor (Fig. 2). Cuando las variables diferencia no presentaron distribución normal de los errores, se transformaron las variables originales y posteriormente se calculó la diferencia (con renoval - sin renoval). Para las variables que no cumplieron el supuesto de homogeneidad de varianzas se modeló la varianza con la función identidad para el factor heterocedástico (Di Rienzo et al., 2017c; Tabla 1).

Posteriormente, para facilitar la interpretación de los resultados, se describió la variación de las condiciones de micrositio según la densidad de ganado y la distancia al borde del bosque, sin distinguir entre cuadrantes con y sin renoval. Para ello se emplearon MLGM con las variables de micrositio como respuesta, la densidad de ganado, la distancia al borde del bosque y la interacción densidad de ganado $\times$ distancia al borde del bosque como factores de efectos fijos y el parche de bosque como factor de efecto aleatorio. La cobertura de vegetación, el porcentaje de suelo desnudo y el porcentaje de roca fueron modelados con distribución Binomial Negativa, ya que fueron registrados como variables discretas y presentaron gran cantidad de ceros y media menor a la varianza. La altura de vegetación, la compactación del suelo y la luminosidad fueron modeladas con distribución Gamma, dado que las tres variables presentaban distribuciones asimétricas. En todos los casos se seleccionó la distribución de probabilidad que minimizara el AIC del modelo y se evaluó el ajuste de forma gráfica (Bolker et al., 2008). La pendiente se analizó empleando distribución Normal por presentar ajuste adecuado y varianza homocedástica.

La cobertura de vegetación fue registrada discriminando entre gramíneas y dicotiledóneas herbáceas, árboles y arbustos, helechos, musgos y vegetación muerta en pie, pero dado que todas las clases de vegetación mostraban el mismo patrón de resultados, se utilizó la cobertura total (sumatoria). También se registró la cobertura de broza, pero no fue analizada por presentar una fuerte correlación con la cobertura de vegetación

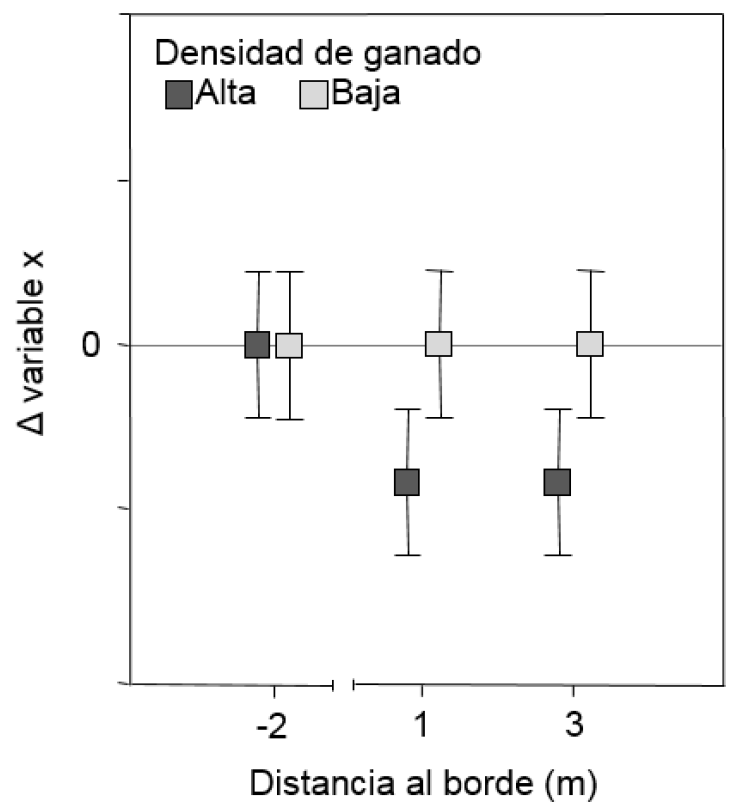

Fig. 2. Gráfico hipotético de una variable diferencia que presenta un efecto de la interacción densidad de ganado $\times$ distancia al borde del bosque. Se muestran medias e intervalos de confianza del $95 \%$ en función de la distancia al borde del bosque ante densidad de ganado alta y baja. Dentro del bosque (a $-2 \mathrm{~m}$ del borde) y para ambas densidades de ganado, los cuadrantes con y sin renoval muestran valores similares, por lo que la diferencia media (con - sin) es igual a cero. Fuera del bosque (a $1 \mathrm{~m}$ y $3 \mathrm{~m}$ del borde), el patrón varía según la densidad de ganado. Ante densidad de ganado alta, los cuadrantes con renoval presentan valores menores que los cuadrantes sin renoval, por lo que la diferencia media es negativa y significativamente distinta de cero (el intervalo de confianza no incluye este valor). En cambio, ante densidad de ganado baja no se encuentra diferencia entre los cuadrantes con y sin renoval.

( $\mathrm{r}$ de Spearman, $\mathrm{r}_{\mathrm{s}}=-0,75 ; \mathrm{p}$-valor, $\mathrm{p}<0,01$ ). La diferencia en luminosidad presentó una distribución aproximadamente uniforme, por lo que fue analizada con una prueba de Kruskal-Wallis para cada factor (densidad de ganado, distancia al borde del bosque e interacción densidad de ganado $x$ distancia al borde del bosque).

Tanto en el análisis de densidad de renovales e intensidad de ramoneo como en el análisis del nicho 
de regeneración, ninguna variable respuesta mostró un efecto significativo del tamaño del parche de bosque, por lo que el mismo no fue incluido en los modelos. Todas las comparaciones múltiples de medias fueron realizadas con la prueba a posteriori LSD Fisher y en todos los análisis se consideró un nivel de significación de 0,05 . Se utilizó el software estadístico InfoStat (Di Rienzo et al., 2017a).

Cabe mencionar que la corta distancia entre los cuadrantes con y sin renoval comparados en el análisis de nicho de regeneración minimiza la diferencia en lluvia de semillas (que puede tener un marcado efecto sobre la ocurrencia de renovales) y permite descontar la variabilidad de las condiciones de micrositio que se observa a mayor escala (es decir, entre parches de bosque, distancias al borde del bosque y potreros con distinta densidad de ganado). De esta manera, aunque la ausencia de renovales en un cuadrante no sea evidencia de que ese micrositio es desfavorable para el establecimiento, una diferencia media distinta de cero entre cuadrantes con y sin renoval sugiere un posible efecto de las condiciones de micrositio sobre la ocurrencia de renovales. Por otro lado, cuando la densidad de renovales es baja, el análisis de micrositios con y sin renovales resulta más conveniente que la metodología tradicional que utiliza micrositios seleccionados al azar, ya que esta última arroja un gran esfuerzo de muestreo en micrositios sin renovales (ver Torres et al., 2008).

\section{Resultados}

\section{Nicho de regeneración}

Sin distinguir entre cuadrantes con y sin renoval, la cobertura y altura de vegetación fueron mayores fuera del bosque (a $1 \mathrm{~m}$ y $3 \mathrm{~m}$ del borde) que dentro (a -2 $\mathrm{m}$ del borde), sin variar según la densidad de ganado (Apéndices 1 y 2). En todos los niveles de densidad de ganado y distancia al borde del bosque, los cuadrantes con renoval presentaron una cobertura de vegetación $18,6 \%$ mayor y una altura de vegetación $68,1 \%$ mayor que los cuadrantes sin renoval (cobertura: Wilcoxon, $Z=3,24 ; p<0,01$; altura: $Z=4,40$; $p$ $<0,01$; Fig. 3A). La cobertura de vegetación fue dominada por gramíneas y dicotiledóneas herbáceas. Las otras clases de vegetación (árboles y arbustos, helechos, musgos y vegetación muerta en pie) mostraron coberturas bajas.
El porcentaje de suelo desnudo fue mayor ante alta densidad de ganado, sin variar según la distancia al borde del bosque (Apéndices 1 y 2). Ante alta densidad de ganado los cuadrantes con renoval presentaron un 5,5\% de suelo desnudo y los cuadrantes sin renoval un $11,4 \%$, mientras que ante baja densidad de ganado los cuadrantes con y sin renoval no difirieron significativamente, presentando un porcentaje bajo (media $=4,1 \%$; Fig. 3B). La diferencia en porcentaje de suelo desnudo (con - sin renoval) no presentó efectos significativos de la distancia al borde del bosque ni la interacción densidad de ganado $\times$ distancia al borde del bosque (Tabla 2).

La compactación del suelo fue mayor ante alta densidad de ganado y fuera del bosque (Apéndices 1 y 2). Dentro del bosque, independientemente de la densidad de ganado, se encontró un bajo grado de compactación, sin diferencias entre cuadrantes con y sin renoval. Sin embargo, fuera del bosque, ante un mayor grado de compactación, los cuadrantes con renoval presentaron una compactación 51,8 \% menor que los cuadrantes sin renoval. A su vez, esta diferencia entre cuadrantes con y sin renoval fue más marcada ante alta densidad de ganado (Fig. 3C, Tabla 2).

La pendiente y el porcentaje de roca no variaron según la densidad de ganado ni la distancia al borde del bosque. En todos los niveles de densidad de ganado y distancia al borde del bosque la pendiente fue un $13,4 \%$ mayor en los cuadrantes con renoval que en los cuadrantes sin renoval $(Z=2,03 ; p=$ $0,04)$, mientras que el porcentaje de roca no mostró diferencias entre los cuadrantes con y sin renoval ( $Z$ $=0,66 ; p=0,51)$. La luminosidad fue menor dentro del bosque y no varió según la densidad de ganado, sin presentar diferencias entre los cuadrantes con y sin renoval en ningún nivel de densidad de ganado ni distancia al borde del bosque $(Z=-0,96 ; p=0,34$; Fig. 3A, Tabla 2, Apéndices 1 y 2).

\section{Densidad de renovales e intensidad de ramoneo}

En el muestreo por transectas se registraron 941 renovales de $S$. commersoniana en un área de $630 \mathrm{~m}^{2}$ y se estimó que el $96,9 \%$ fue originado por semilla y el 3,1 \% agámicamente por raíz. La densidad de renovales fue mayor con baja densidad de ganado $\left(2,07 \pm 0,23\right.$ renovales. $\mathrm{m}^{-2}$; media \pm error estándar) que con alta densidad de ganado $(1,86$ $\pm 0,38$ renovales. $\mathrm{m}^{-2}$; Fisher, $\mathrm{F}=4,68 ; \mathrm{p}=0,03$ ) 


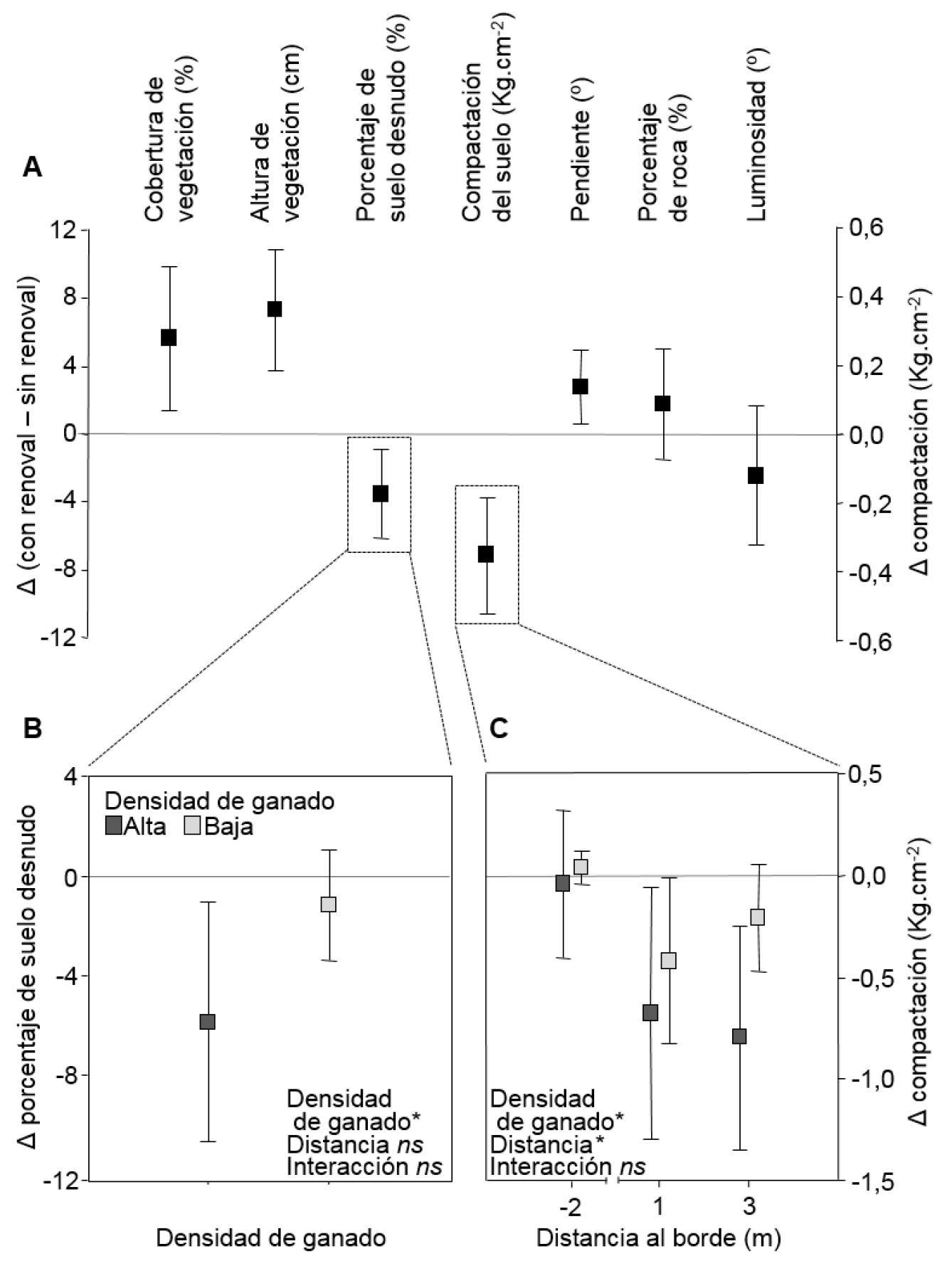

Fig. 3. A. Diferencia media (con renoval - sin renoval) e intervalo de confianza del $95 \%$ para las variables de micrositio. Cuando el intervalo de confianza de una variable no cruza la línea de cero, los cuadrantes con renoval difieren significativamente de los cuadrantes sin renoval. B. Diferencia media en porcentaje de suelo desnudo en función de la densidad de ganado. C. Diferencia media en compactación del suelo en función de la distancia al borde del bosque ante densidad de ganado alta y baja. Se indica con un asterisco $\left.{ }^{*}\right)$ a los valores de $p \leq 0,05$ y con "ns" a los valores de $p>0,05$. 
Tabla 2. Efectos de la densidad de ganado, la distancia al borde del bosque y la interacción densidad de ganado $\times$ distancia al borde del bosque sobre las variables diferencia (con renoval - sin renoval). Se indican con un asterisco $\left(^{*}\right)$ las variables con media significativamente distinta de cero (ver Fig. $3 A$ ); F = estadístico F de Fisher; $\mathrm{H}$ = estadístico de la prueba de Kruskal-Wallis; $\mathrm{p}$ = p-valor.

\begin{tabular}{|c|c|c|c|c|c|c|}
\hline \multirow{3}{*}{ Variable diferencia } & \multicolumn{6}{|c|}{ Factores analizados } \\
\hline & \multicolumn{2}{|c|}{ Densidad de ganado } & \multicolumn{2}{|c|}{ Distancia al borde } & \multicolumn{2}{|c|}{ Interacción } \\
\hline & $\mathrm{F}$ & $\mathrm{p}$ & $\mathrm{F}$ & $\mathrm{p}$ & $\mathrm{F}$ & $\mathrm{p}$ \\
\hline${ }^{*}$ Cobertura de vegetación & $<0,01$ & 0,96 & 1,66 & 0,20 & 1,16 & 0,32 \\
\hline${ }^{*}$ Altura de vegetación & 1,07 & 0,31 & 1,18 & 0,32 & 2,24 & 0,11 \\
\hline 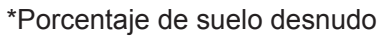 & 4,03 & 0,05 & 2,09 & 0,13 & 1,98 & 0,15 \\
\hline${ }^{*}$ Compactación del suelo & 3,92 & 0,05 & 3,78 & 0,03 & 1,01 & 0,37 \\
\hline *Pendiente & 0,08 & 0,77 & 0,24 & 0,79 & 1,95 & 0,15 \\
\hline \multirow[t]{2}{*}{ Porcentaje de roca } & 0,13 & 0,57 & 0,19 & 0,22 & 1,32 & 0,30 \\
\hline & $\mathrm{H}$ & $\mathrm{p}$ & $\mathrm{H}$ & $\mathrm{p}$ & $\mathrm{H}$ & $\mathrm{p}$ \\
\hline Luminosidad & 1,13 & 0,73 & 1,53 & 0,83 & 6,05 & 0,27 \\
\hline
\end{tabular}

y en ambas situaciones disminuyó al aumentar la distancia al borde del bosque $(F=25,69 ; p<0,01)$. Además, se encontró un efecto significativo de la interacción densidad de ganado $\times$ distancia al borde del bosque $(\mathrm{F}=8,19 ; \mathrm{p}<0,01)$ : dentro del bosque la densidad de renovales no varió según la densidad de ganado, pero sí varió fuera del bosque (Fig. 4A).

La proporción de tallos ramoneados ante alta densidad de ganado fue 13 veces mayor que ante baja densidad de ganado ( $F=71,60 ; p<0,01$; Fig. 4B). Dentro del bosque los renovales se encontraron más ramoneados que fuera, mostrando mayor diferencia ante alta densidad de ganado (distancia al borde del bosque: $F=2,91 ; p=0,05$ ) y no se encontró efecto significativo de la interacción densidad de ganado $\times$ distancia al borde del bosque $(\mathrm{F}=1,84 ; \mathrm{p}=$ 0,16; Fig. 4B). La altura de los renovales tuvo un efecto significativo sobre la proporción de tallos ramoneados, encontrándose más ramoneados los renovales más altos $(\mathrm{F}=66,07 ; \mathrm{p}<0,01)$.

\section{Discusıón}

Nuestros resultados sugieren que los renovales de $S$. commersoniana se encuentran en micrositios con abundante vegetación, poco suelo desnudo, baja compactación del suelo y elevada pendiente.
Este patrón no varió en función de la densidad de ganado y la distancia al borde del bosque. Sin embargo, no podemos descartar que dicha falta de variación se deba a que los potreros comparados no hayan presentado una diferencia notoria en densidad de ganado (o a que haya transcurrido poco tiempo desde que se redujo la densidad de ganado en uno de los potreros) y a que las distancias al borde del bosque analizadas hayan sido muy cortas. La ocurrencia de renovales en micrositios con abundante vegetación independientemente de la densidad de ganado coincide con lo esperado bajo la hipótesis de predominancia de facilitación directa. Esto concuerda en parte con estudios realizados con otras especies arbóreas del Bosque Serrano de Córdoba, donde Torres y Renison (2015, 2016) encontraron facilitación directa e indirecta (mediada por herbívoros) por parte de árboles y arbustos. No obstante, nuestros resultados no coinciden con lo esperado bajo facilitación directa mediada por disminución del estrés hídrico, ya que fuera del bosque no se encontró una asociación más notoria entre renovales y micrositios con abundante vegetación herbácea. Esto puede deberse a que el estrés hídrico no varíe marcadamente entre las distancias al borde del bosque evaluadas, a que el mecanismo de facilitación no sea la disminución del estrés hídrico o a que, dentro del bosque, 
A

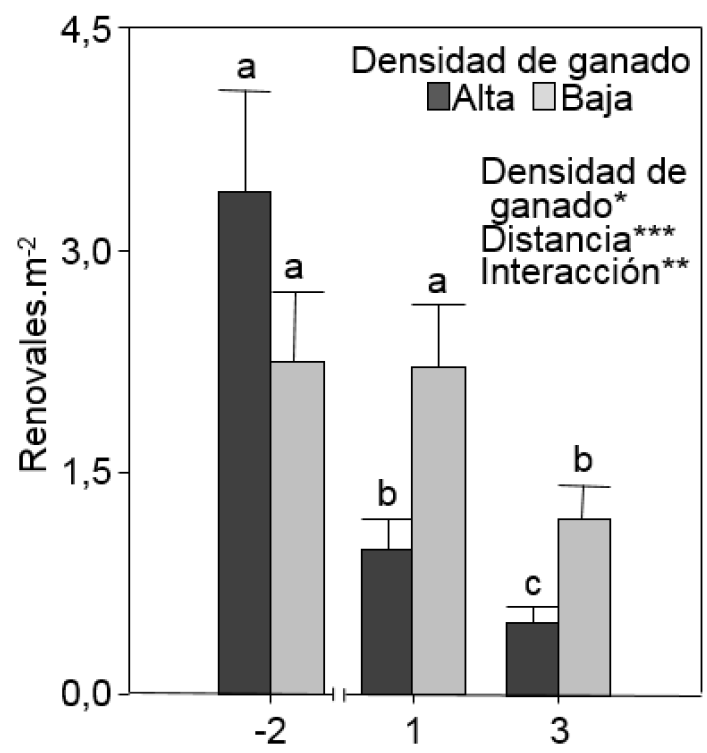

B

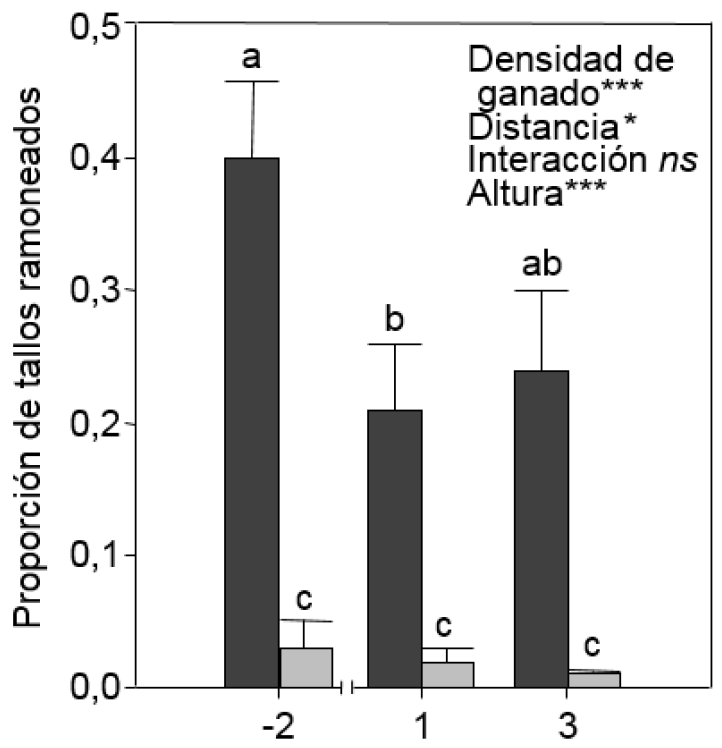

Distancia al borde $(\mathrm{m})$

Fig. 4. A. Densidad media de renovales en función de la distancia al borde del bosque ante densidad de ganado alta y baja. B. Proporción media de tallos ramoneados en función de la distancia al borde del bosque ante densidad de ganado alta y baja. Se muestran barras de error estándar. Letras distintas indican medias significativamente diferentes. Se indica con uno, dos y tres asteriscos $\left({ }^{*}\right)$ a los valores de $p \leq 0,05, p$ $<0,001$ y $p<0,0001$, respectivamente, y se indican con " $n s$ " los valores de $p>0,05$.

otro factor (e.g., disponibilidad de nutrientes) favorezca tanto a la vegetación herbácea como a los renovales. En cuanto a las condiciones del suelo, nuestros resultados coinciden con los de Torres y colaboradores (2008), quienes encontraron que los renovales de una especie arbórea del Bosque Serrano de Córdoba se hallaban en micrositios poco degradados por el ganado.

La ocurrencia de renovales de $S$. commersoniana en micrositios con abundante vegetación y suelo poco degradado podría deberse a que estas condiciones favorezcan la regeneración. Tanto la germinación como la supervivencia de renovales podrían aumentar ante la disminución del estrés hídrico, atenuación de extremos de temperatura y/o facilitación de la penetración de las raíces en el suelo (Castellano \& Valone, 2007; Sinnett et al., 2008; Urbieta et al., 2008; Venier et al., 2013). Además, la vegetación podría actuar como trampa de semillas arrastradas por escorrentía. Sin embargo, la ocurrencia de renovales en micrositios con elevada pendiente se contrapone parcialmente a estas interpretaciones. Esta asociación podría explicarse en parte por la menor compactación que presentan los micrositios más inclinados (por ser menos pisoteados por el ganado; $r_{s}=-0,25 ; p$ $<0,01)$, pero a mayor pendiente se esperaría menor contenido de humedad del suelo (Fox et al., 1997; Thierfelder \& Wall, 2009) y menor permanencia de semillas debido a la mayor escorrentía. Ante este escenario, cabe mencionar que los patrones observados pueden explicarse por diversos procesos; por lo tanto, se requiere de un abordaje experimental para avanzar en el entendimiento de los efectos que tienen las condiciones de micrositio sobre la regeneración.

Con respecto a la densidad de renovales, nuestros resultados coinciden con numerosos estudios que sugieren efectos negativos del ganado doméstico sobre la regeneración temprana de 
especies leñosas (Dufour-Dror, 2007; Torres et al., 2008; Plieninger et al., 2011), encontrando menor densidad de renovales ante mayor densidad de ganado. Esto podría explicarse tanto por efectos directos del ganado sobre los renovales, como ramoneo y pisoteo, como por efectos indirectos: dado que el ganado disminuye la biomasa del estrato herbáceo y degrada el suelo (Yates et al., 2000; Chaichi et al., 2005), el mismo podría dificultar la regeneración de la especie (en base a nuestro análisis del nicho de regeneración). No obstante, encontramos que dentro del bosque la densidad de renovales no varió según la densidad de ganado, a pesar de que la proporción de tallos ramoneados fue mayor dentro del bosque y ante alta densidad de ganado. Por un lado, es posible que dentro del bosque los efectos indirectos del ganado sobre los renovales sean menos notorios debido a que la vegetación herbácea y el suelo están menos expuestos a factores externos como la insolación, la lluvia, el viento y las heladas (Murcia, 1995). Por otro lado, es posible que el ramoneo, que probablemente retarde el crecimiento, no afecte tanto la supervivencia de renovales por tratarse de una especie rebrotante (Drexhage \& Colin, 2003). Asimismo, la disminución en densidad de renovales observada a pocos metros fuera del bosque sugiere que, a mayores distancias, el reclutamiento puede verse limitado por semillas, teniendo en cuenta la baja capacidad de dispersión que presenta la especie (Holl, 1999; Cubiña \& Aide, 2001). Aun así, esto debe verificarse evaluando la importancia de la dispersión secundaria.

Concluimos que el establecimiento de renovales de $S$. commersoniana puede verse favorecido en micrositios conservados en cuanto a la vegetación y el suelo; que el reclutamiento de renovales puede encontrarse limitado a las cercanías de los parches de bosque, y que la reducción en densidad de ganado puede favorecer la regeneración de la especie. Estos resultados pueden ser de utilidad para la conservación y manejo, dado que la presencia de ganado doméstico es frecuente en las áreas donde se distribuye esta especie, por lo cual su regeneración podría encontrarse limitada. Sugerimos aprovechar futuras oportunidades de comparar usos ganaderos contrastantes para avanzar en el entendimiento de los patrones aquí expuestos mediante un acercamiento experimental y una evaluación más detallada de las condiciones microambientales.

\section{Agradecimientos}

Agradecemos a Ana M. Cingolani por su asesoramiento en el análisis de datos; a Guillermo Funes, Leonardo Galetto, Juan Manuel Rodríguez y dos revisores anónimos por sus valiosos comentarios sobre el manuscrito; a Leonardo Barberá y Federico Guevara por su colaboración en los muestreos, y a las familias Renison-Cingolani y Gonzáles por los permisos para realizar los muestreos y la información brindada sobre densidades de ganado. El estudio se financió con fondos del programa de cooperación bilateral - Nivel II (PCB-II) MINCyT - CONICET - DFG para el proyecto "Efectos combinados de la altitud, el fuego y las interacciones bióticas en la regeneración de especies arbóreas en una montaña subtropical del Centro Argentino".

\section{Bibliografía}

BAIER, R., J. MEYER \& A. GÖTTLEIN. 2007. Regeneration niches of Norway spruce (Picea abies [L.] Karst.) saplings in small canopy gaps in mixed mountain forests of the Bavarian Limestone Alps. Eur. J. For. Res. 126: 11-22.

BARTUSZEVIGE, A. M. \& B. A. ENDRESS. 2008. Do ungulates facilitate native and exotic plant spread? Seed dispersal by cattle, elk and deer in Northeastern Oregon. J. Arid Environ. 72: 904-913.

BOLKER, B. M., M. E. BROOKS, C. J. CLARK, S. W. GEANGE, J. R. POUlSEN, M. H. H. STEVENS \& J. S. S. WHITE. 2009. Generalized linear mixed models: a practical guide for ecology and evolution. Trends Ecol. Evol. 24: 127-135.

CABRERA, A. L. 1971. Fitogeografía de la república Argentina. Bol. Soc. Argent. Bot. 14: 1-42.

CASTELLANO, M. J. \& T. J. VALONE. 2007. Livestock, soil compaction and water infiltration rate: Evaluating a potential desertification recovery mechanism. J. Arid Environ. 71: 97-108.

CAYLOR, K. K., H. H. SHUGART \& I. RODRIGUEZITURBE. 2005. Tree canopy effects on simulated water stress in southern African savannas. Ecosystems. 8: 17-32.

CHAICHI, M. R., M. M. SARAVI \& A. MALEKIAN. 2005. Effects of livestock trampling on soil physical properties and vegetation cover (case study: Lar Rangeland, Iran). Int. J. Agric. Biol. 7: 904-908.

CHRISTIE, D. A. \& J. J. ARMESTO. 2003. Regeneration microsites and tree species coexistence in temperate rain forests of Chiloé Island, Chile. J. Ecol. 91: 776784. 
COLlADON, L. \& I. PAZOS. 2014. Anuario pluviométrico 1992/93-2011/12. Cuenca del Río San Antonio. Sistema del Río Suquía-Provincia de Córdoba. Instituto Nacional del Agua y del Ambiente (INA) y Centro de Investigaciones de la Región Semiárida (CIRSA), Córdoba.

COLlin, A., C. MESSIER, S. W. KEMBEL \& N. BÉLANGER. 2017. Low light availability associated with American beech is the main factor for reduced sugar maple seedling survival and growth rates in a hardwood forest of Southern Quebec Forests 8 (11): 1-13.

CUBIÑA, A. \& M. T. AIDE. 2001. The effect of distance from forest edge on seed rain and soil seed bank in a tropical pasture.Biotropica 33: 260-267.

DAVIES-COLLEY, R. J., G. W. PAYNE \& M. VAN ELSWIJK. 2000. Microclimate gradients across a forest edge. New Zeal. J. Ecol. 24: 111-121.

DEMAIO, P., U. KARLIN \& M. MEDINA. 2002. Árboles nativos del centro de Argentina. LOLA, Buenos Aires.

DE SOUZA, I. F., A. F. SOUZA, M. A. PIZO \& G. GANADE. 2010. Using tree population size structures to assess the impacts of cattle grazing and eucalypts plantations in subtropical South America. Biodivers. Conserv. 19: 1683-1698.

DI RIENZO, J. A., F. CASANOVES, M. G. BALZARINI, L. GONZALEZ, M. TABLADA \& C. W. ROBLEDO. 2017a. InfoStat. Córdoba, Argentina. Grupo InfoStat. Disponible en: http:// www.infostat.com.ar

DI RIENZO, J. A., E. MACCHIAVELLI \& F. CASANOVES. 2017b. Modelos lineales generalizados mixtos: aplicaciones en InfoStat. Grupo InfoStat, Córdoba.

DI RIENZO, J. A., E. MACCHIAVELLI \& F. CASANOVES. 2017c. Modelos lineales mixtos: aplicaciones en InfoStat. Grupo InfoStat, Córdoba.

DREXHAGE, M. \& F. COLIN. 2003. Effects of browsing on shoots and roots of naturally regenerated sessile oak seedlings. Ann. For. Sci. 60: 173-178.

DUFOUR-DROR, J. M. 2007. Influence of cattle grazing on the density of oak seedlings and saplings in a Tabor oak forest in Israel. Acta Oecol. 31: 223-228.

DUNCAN, R. S. \& V. E. DUNCAN. 2000. Forest succession and distance from forest edge in an Afro-tropical grassland. Biotropica 32: 33-41.

FENNER, M. \& K. THOMPSON. 2005. The ecology of seeds. 2nd Ed. Cambridge University Press, Cambridge.

FILAZZOLA, A. \& C. J. LORTIE. 2014. A systematic review and conceptual framework for the mechanistic pathways of nurse plants. Global Ecol. Biogeogr. 23: 1335-1345.
FLORES, J. \& E. JURADO. 2003. Are nurse-protégé interactions more common among plants from arid environments? J. Veg. Sci. 14: 911-916.

FOX, D. M., R. B. BRYAN \& A. G. PRICE. 1997. The influence of slope angle on final infiltration rate for interrill conditions. Geoderma 80: 181-194.

GIORGIS, M. A, A. M CINGOLANI, F. CHIARINI, J. CHIAPELLA, G. BARBOZA, L. ARIZA ESPINAR, R. MORERO, D. E. GURVICH, P. A. TECCO, R. SUBILS \& M. CABIDO. 2011. Composición florística del Bosque Chaqueño Serrano de la provincia de Córdoba, Argentina. Kurtziana 36: 9-43.

GÓMEZ-APARICIO, L. 2009. The role of plant interactions in the restoration of degraded ecosystems: A meta-analysis across life-forms and ecosystems. $J$. Ecol. 97: 1202-1214.

GOSWAMI, D. Y., F. KREITH \& J. F. KREIDER. 2000. Principles of solar engineering. 2nd ed. Taylor \& Francis, Philadelphia.

GRISCOM, H. P., B. W. GRISCOM \& M. S. ASHTON. 2009. Forest regeneration from pasture in the dry tropics of Panama: Effects of cattle, exotic grass, and forested riparia. Restor. Ecol. 17: 117-126.

HANSEN, M. C., P. V. POTAPOV, R. MOORE, M. HANCHER, S. A. TURUBANOVA, A. TYUKAVINA, D. THAU, S. V. STEHMAN, S. J. GOETZ, T. R. LOVELAND, A. KOMMAREDDY, A. EGOROV, L. CHINI, C. O. JUSTICE \& J. R. G. TOWNSHEND. 2013. High-resolution global maps of 21st-century forest cover change. Science 342: 850-854.

HEINEMANN, K., T. KITZBERGER \& T. T. VEBLEN. 2000. Influences of gap microheterogeneity on the regeneration of Nothofagus pumilio in a xeric old-growth forest of northwestern Patagonia, Argentina. Canad. J. Forest Res. 30: 25-31.

HOLL, K. D. 1999. Factors limiting tropical rain forest regeneration in abandoned pasture: seed rain, seed germination, microclimate, and soil. Biotropica 31: 229-242.

HOLMGREN, M., M. SCHEFFER \& M. A. HUSTON. 1997. The interplay of facilitation and competition in plant communities. Ecology78: 1966-1975.

KELLNER, K. F. \& R. K. SWIHART. 2016. Timber harvest and drought interact to impact oak seedling growth and survival in the Central Hardwood Forest. Ecosphere 7: 1-15.

KHISHIGJARGAL, M., C. DULAMSUREN, D. LKHAGVADORJ, C. LEUSCHNER \& M. HAUCK. 2013. Contrasting responses of seedling and sapling densities to livestock density in the Mongolian foreststeppe. Plant Ecol. 214: 1391-1403.

MEDEIROS, A. D. S. \& A. ZANON. 1998. Conservação de sementes de branquilho (Sebastiania commersoniana (Baillon) LB Smith \& RJ Down) 
e de pinheiro-bravo (Podocarpus lambertii Klotzch ex e NDL.) armazenadas em diferentes ambientes. Boletim Pesq. Flor.36: 57-69.

MEINERS, S. J. \& M. J. MARTINKOVIC. 2002. Survival of and herbivore damage to a cohort of Quercus rubra planted across a forest-old-field edge. ґAmer. Midl. Nat. 147: 247-255.

MUNIER, A., L. HERMANUTZ, J. D. JACOBS \& K. LEWIS. 2010. The interacting effects of temperature, ground disturbance and herbivory on seedling establishment: implications for treeline advance with climate warming. Plant Ecol. 210: 19-30.

MURCIA, C. 1995. Edge effects in fragmented forests: implications for conservation. Trends Ecol. Evol. 10: 58-62.

O'HARA, R. B. \& D. J. KOTZE. 2010. Do not logtransform count data. Methods Ecol. Evol. 1: 118122.

PIAGGiO, M. \& L. DELFINO. 2009. Florística y fitosociología de un bosque fluvial en Minas de Corrales, Rivera, Uruguay. Iheringia 64: 45-51.

PLIENINGER, T., H. SCHAICH \& T. KIZOS. 2011. Landuse legacies in the forest structure of silvopastoral oak woodlands in Eastern Mediterranean. Reg. Environ. Change 11: 603-615.

POORTER, L. 2007. Are species adapted to their regeneration niche, adult niche, or both? Amer. Naturalist. 169: 433-442.

RENISON, D., I. HENSEN \& A. M. CINGOLANI. 2004. Anthropogenic soil degradation affects seed viability in Polylepis australis mountain forests of central Argentina. Forest Ecol. Manag. 196: 327-333.

SINNETT, D., G. MORGAN, M. WILLIAMS \& T. R. HUTCHINGS. 2008. Soil penetration resistance and tree root development. Soil Use Manage. 24: 273-280.

THIERFELDER, C. \& P. C. WALL. 2009. Effects of conservation agriculture techniques on infiltration and soil water content in Zambia and Zimbabwe. Soil Till. Res. 105: 217-227.

TORRES, R. C. \& D. RENISON. 2015. Effects of vegetation and herbivores on regeneration of two tree species in a seasonally dry forest. J. Arid Environ. 121: 59-66.

TORRES, R. C. \& D. RENISON. 2016. Indirect facilitation becomes stronger with seedling age in a degraded seasonally dry forest. Acta Oecol. 70: 138-143.
TORRES, R.C., D. RENISON, I.HENSEN, R. SUAREZ\& L. ENRICO. 2008. Polylepis australis' regeneration niche in relation to seed dispersal, site characteristics and livestock density. Forest Ecol. Manag. 254: 255-260.

URBIETA, I. R., I. M. PÉREZ-RAMOS, M. A. ZAVALA, T. MARAÑ́́N \& R. K. KOBE. 2008. Soil water content and emergence time control seedling establishment in three co-occurring Mediterranean oak species. Canad. J. Forest Res. 38: 2382-2393.

VALFRÉ-GIORELLO, T. A., L. ASHWORTH \& D. RENISON. 2012. Patrones de germinación de semillas de Sebastiania commersoniana (Baillon) Smith \& Downs (Euphorbiaceae), árbol nativo del Chaco Serrano de interés en restauración. Ecol. Austral 22: 92-100.

VENIER, P., M. CABIDO, A. MANGEAUD \& G. FUNES. 2013. Crecimiento y supervivencia de renovales de cinco especies de Acacia (Fabaceae), que coexisten en bosques secos neotropicales de Argentina, en distintas condiciones de disponibilidad de luz y agua.Revista Biol. Trop. 61: 501-514.

VINCENT, A. \& S. J. DAVIES. 2003. Effects of nutrient addition, mulching and planting-hole size on early performance of Dryobalanops aromatica and Shorea parvifolia planted in secondary forest in Sarawak, Malaysia. Forest Ecol. Manag. 180: 261-271.

VON MÜller, A. R., A. M. CINGOLANI, M. V. VAIERETTI \& D. RENISON. 2012. Estimación de carga bovina localizada a partir de frecuencia de deposiciones en un pastizal de montaña. Ecol. Austral 22: 178-187.

YATES, C. J., D. A. NORTON \& R. J. HOBBS. 2000. Grazing effects on plant cover, soil and microclimate in fragmented woodlands in south-western Australia: implications for restoration. Austral Ecol. 25: 36-47.

ZAK, M. R., M. CABIDO \& J. G. HODGSON. 2004. Do subtropical seasonal forests in the Gran Chaco, Argentina, have a future? Biol. Conserv. 120: 589598.

Recibido el 26 de enero de 2018, aceptado el 9 de mayo de 2018. Editor: Guillermo Funes. 


\section{Barberá et al. - Pastoreo y regeneración de Sebastiania commersoniana}

Apéndice 1. Media y error estándar de las variables de micrositio para densidad de ganado alta y baja y en las tres distancias al borde del bosque, sin diferenciar cuadrantes con y sin renoval $(n=36)$. Se indica con un asterisco $\left(^{*}\right)$ a las variables con diferencia media (con renoval - sin renoval) significativamente distinta de cero (ver Fig. 3A). Letras distintas indican diferencias significativas.

\begin{tabular}{|c|c|c|c|c|}
\hline \multirow{2}{*}{ Variable } & \multirow{2}{*}{$\begin{array}{c}\text { Densidad de } \\
\text { ganado }\end{array}$} & \multicolumn{3}{|c|}{ Distancia al borde (m) } \\
\hline & & -2 & 1 & 3 \\
\hline \multirow{2}{*}{${ }^{*}$ Cobertura de vegetación (\%) } & Alta & $10,5 \pm 2,1^{a}$ & $37,5 \pm 3,7^{b}$ & $37,5 \pm 3,6^{b}$ \\
\hline & Baja & $12,8 \pm 2,1^{a}$ & $46 \pm 4,0^{b}$ & $52,7 \pm 4,7^{b}$ \\
\hline \multirow{2}{*}{ *Altura de vegetación (cm) } & Alta & $4,5 \pm 1,1^{a}$ & $14,9 \pm 2,3^{b}$ & $21,2 \pm 3,8^{b}$ \\
\hline & Baja & $6,9 \pm 1,4^{a}$ & $18,1 \pm 1,7^{b}$ & $20,7 \pm 2,0^{b}$ \\
\hline \multirow{2}{*}{${ }^{*}$ Porcentaje de suelo desnudo (\%) } & Alta & $8,0 \pm 2,3^{b c}$ & $6,7 \pm 1,4^{\mathrm{bc}}$ & $10,6 \pm 2,4^{c}$ \\
\hline & Baja & $5,5 \pm 1,5^{a b}$ & $3,4 \pm 0,8^{a}$ & $3,2 \pm 0,8^{a}$ \\
\hline \multirow{2}{*}{${ }^{\star}$ Compactación del suelo $\left(\mathrm{Kg} \cdot \mathrm{cm}^{-2}\right)$} & Alta & $0,54 \pm 0,10^{b}$ & $0,99 \pm 0,16^{c}$ & $0,92 \pm 0,16^{c}$ \\
\hline & Baja & $0,21 \pm 0,03^{a}$ & $0,51 \pm 0,11^{\mathrm{b}}$ & $0,44 \pm 0,09^{b}$ \\
\hline \multirow{2}{*}{${ }^{*}$ Pendiente $\left({ }^{\circ}\right)$} & Alta & $21,3 \pm 1,7^{a}$ & $20,6 \pm 1,6^{a}$ & $20,8 \pm 1,7^{a}$ \\
\hline & Baja & $22,3 \pm 2,2^{a}$ & $24,1 \pm 2,2^{a}$ & $23,2 \pm 1,8^{a}$ \\
\hline \multirow{2}{*}{ Porcentaje de roca (\%) } & Alta & $9,7 \pm 2,6^{a}$ & $10,1 \pm 1,9^{a}$ & $9,7 \pm 2,4^{a}$ \\
\hline & Baja & $11,5 \pm 2,0^{a}$ & $10,2 \pm 2,3^{a}$ & $7,1 \pm 1,8^{a}$ \\
\hline \multirow{2}{*}{ Luminosidad $\left({ }^{\circ}\right)$} & Alta & $35,3 \pm 3,5^{a}$ & $118,4 \pm 4,6^{b}$ & $120,5 \pm 5,2^{b}$ \\
\hline & Baja & $28,6 \pm 3,5^{a}$ & $105,1 \pm 5,7^{b}$ & $118,1 \pm 5,0^{b}$ \\
\hline
\end{tabular}


Bol. Soc. Argent. Bot. 53 (3) 2018

Apéndice 2, Efectos de la densidad de ganado, la distancia al borde del bosque y la interacción densidad de ganado $\times$ distancia al borde del bosque sobre las variables de micrositio (sin diferenciar cuadrantes con y sin renoval), Se indica con un asterisco $\left({ }^{*}\right)$ a las variables con diferencia media (con renoval - sin renoval) significativamente distinta de cero (ver Fig, 3A),

\begin{tabular}{|c|c|c|c|c|c|c|}
\hline \multirow{3}{*}{ Variable } & \multicolumn{6}{|c|}{ Factores analizados } \\
\hline & \multicolumn{2}{|c|}{ Densidad de ganado } & \multicolumn{2}{|c|}{ Distancia al borde } & \multicolumn{2}{|c|}{ Interacción } \\
\hline & $\mathrm{F}$ & $\mathrm{p}$ & $\mathrm{F}$ & $\mathrm{p}$ & $\mathrm{F}$ & $\mathrm{p}$ \\
\hline${ }^{*}$ Cobertura de vegetación & 2,97 & 0,12 & 102,59 & $<0,01$ & 0,08 & 0,93 \\
\hline${ }^{*}$ Altura de vegetación & 1,57 & 0,21 & 36,91 & $<0,01$ & 0,91 & 0,40 \\
\hline 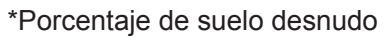 & 10,87 & $<0,01$ & 1,09 & 0,34 & 2,09 & 0,13 \\
\hline *Compactación del suelo & 10,81 & $<0,01$ & 14,4 & $<0,01$ & 0,13 & 0,88 \\
\hline *Pendiente & 0,85 & 0,36 & 0,06 & 0,94 & 0,31 & 0,73 \\
\hline Porcentaje de roca & 0,04 & 0,84 & 0,40 & 0,67 & 0,38 & 0,68 \\
\hline Luminosidad & 0,98 & 0,32 & 311,17 & $<0,01$ & 1,51 & 0,22 \\
\hline
\end{tabular}

